\title{
Clinical Impact of Preexisting Right Bundle Branch Block after Transcatheter Aortic Valve Replacement: A Systematic Review and Meta-Analysis
}

\author{
Garly R. Saint Croix ${ }^{(D},{ }^{1}$ Spencer C. Lacy ${ }^{(D},{ }^{2}$ Hakop Hrachian, ${ }^{1}$ and Nirat Beohar ${ }^{1}$ \\ ${ }^{1}$ Columbia University Division of Cardiology, Mount Sinai Medical Center, Miami Beach, FL, USA \\ ${ }^{2}$ Miller School of Medicine, The University of Miami, Miami, FL, USA \\ Correspondence should be addressed to Garly R. Saint Croix; garly.saintcroix@msmc.com
}

Received 12 April 2020; Revised 20 June 2020; Accepted 30 June 2020; Published 22 July 2020

Academic Editor: Toshiko Nakai

Copyright (C) 2020 Garly R. Saint Croix et al. This is an open access article distributed under the Creative Commons Attribution License, which permits unrestricted use, distribution, and reproduction in any medium, provided the original work is properly cited.

\begin{abstract}
Introduction. Transcatheter aortic valve replacement (TAVR) is now the treatment of choice for patients with severe aortic stenosis regardless of their surgical risk. Right bundle branch block (RBBB) can be a predictor for development of significant atrioventricular (AV) block after TAVR, requiring permanent pacemaker implantation (PPI). However, data related to the risk of PPI requirement with preexisting $\mathrm{RBBB}$ is scarce. Hence, this systematic review and meta-analysis aims to assess clinical outcomes of patients undergoing TAVR with RBBB on preexisting electrocardiogram. Methods. We performed a systematic literature review to identify randomized and nonrandomized clinical studies that reported any clinical impact of patients undergoing TAVR with preexisting RBBB. A total of eight databases including PubMed (Medline), Embase, Cochrane Library, ACP Journal Club, Scopus, DARE, and Ovid containing articles from January 2000 to May 2020 were analyzed. Results. We identified and screened 224 potential eligible publications through the databases and found 14 relevant clinical trials for a total of 15,319 participants. There was an increased 30-day pacemaker implantation rate of $38.1 \%$ in the RBBB group compared to $11.4 \%$ in the no RBBB group with a risk ratio of 3.56 (RR $3.56(95 \%$ CI 3.21-3.93, $p<0.01)$ ). There was an increased 30-day all-cause mortality in the RBBB group of 9.5\% compared with $6.3 \%$ in the no RBBB group with an odds ratio of 1.60 (OR 1.60 (95\% CI 1.14-2.25, p<0.01)). Conclusion. This study indicates that patients with preexisting RBBB have higher incidence of PPI and all-cause mortality after TAVR compared with patients without RBBB. Further trials are needed to compare the clinical outcomes based on TAVR valve types and assess the benefit of PPI in patients with new-onset RBBB after TAVR.
\end{abstract}

\section{Introduction}

Transcatheter aortic valve replacement (TAVR) has revolutionized the current era of modern medicine by becoming the treatment of choice for patients with symptomatic severe aortic stenosis regardless of their surgical risk [1]. However, the high frequency of conduction disturbances, such as left bundle branch block (LBBB) and atrioventricular block, and the subsequent need for permanent pacemaker implantation (PPI) remain a challenge $[2,3]$. Preexisting right bundle branch block (RBBB) has been established as a risk factor for PPI after TAVR $[4,5]$. Preexisting RBBB in the general population and in patients with heart disease has been associated with increased risk of mortality [6]. However, data on the prognostic impact of preexisting RBBB on clinical outcomes after TAVR is limited. This systematic review and meta-analysis evaluates the impact of preexisting RBBB on clinical outcomes in patients undergoing TAVR.

\section{Methods}

The main objective of this review was to assess if preexisting RBBB increased the risk of having PPI after TAVR. We used the Preferred Reporting Items for Systematic Reviews and Meta-Analyses (PRISMA) statement extension for network meta-analysis. The PRISMA flow diagram was used to depict the four phases of the review including identification, screening, eligibility, and inclusion. The PRISMA statement 
contains a checklist of 27 items required of systematic reviews and meta-analyses. The review was not registered a priori. No ethical approval was required since this metaanalysis uses only public published data.

2.1. Search Strategy. We performed a systematic literature review to identify randomized and nonrandomized clinical studies that reported any clinical impact of patients undergoing TAVR with preexisting RBBB. Searches were limited to peer-reviewed primary research articles published in English, French, and Spanish up to May $17^{\text {th }}, 2020$. This research involved human subjects and described the clinical impact of $\mathrm{RBBB}$ on patients who underwent TAVR. We developed the search strategy according to available guidance from the Cochrane Collaboration.

The search strategy in MEDLINE explored Medical Subject Heading (MeSH) terms related to patients with TAVR and history of preexisting RBBB. The following search strategy was applied to search MEDLINE and we adapted it for the other databases: ("transcatheter aortic valve replacement"[MeSH Terms] OR ("transcatheter"[All Fields] AND “aortic"[All Fields] AND "valve"[All Fields] AND “replacement"[All Fields] AND “implantation”[All Fields])) OR "transcatheter aortic valve replacement"[All Fields]) AND ("bundle-branch block"[MeSH Terms] OR ("bundle-branch"[All Fields] AND "block"[All Fields]) OR "bundle-branch block"[All Fields] OR ("right”[All Fields] AND "bundle"[All Fields] AND "branch"[All Fields] AND "block"[All Fields]) OR "right bundle branch block"[All Fields]). The articles found to be relevant during the hand search were stored in EndNote. Selected articles underwent full evaluation to assess their potential inclusion in the systematic review.

2.2. Study Selection. Articles were selected for inclusion based on predefined criteria, which included age, sex, TAVR, and preexisting $\mathrm{RBBB}$, and the primary or secondary outcomes being mortality and clinical outcomes. Exclusion criteria were patients with LBBB and patients with normal sinus rhythm. We excluded case reports and studies with fewer than 10 subjects.

Two authors (GS, SL) independently read the trials and screened the abstracts to choose potentially relevant articles. Risk of bias in the studies was assessed at an individual level of each study. Selected articles underwent full evaluation to assess their potential inclusion in the systematic review.

2.3. Statistical Analysis. Data were analyzed using Review Manager Software 5.3. We used fixed effects to assess the combined risk estimates according to I2 statistics. Analysis to determine sensitivity and publication bias was detected by funnel plots. $p<0.05$ was considered statistically significant.

\section{Results}

3.1. Literature Search. Our search yielded 224 abstracts. We excluded 199 studies at the abstract level and selected 24 full- text articles for detailed assessment; 14 studies were ultimately included in our systematic review and 11 studies were included in our meta-analysis. Figure 1 describes the flowchart of included studies.

3.2. Baseline Characteristics of the Studies. Table 1 shows the baseline characteristics of the included studies. All studies were published between 2010 and 2020. The 14 studies included 15,319 patients with 1,654 cases of preexisting $\mathrm{RBBB}$. In nine of the included studies, preexisting RBBB was retrospectively identified as a risk factor for PPI. Therefore, baseline characteristics for patients with preexisting $\mathrm{RBBB}$ were not reported in these nine studies. For studies that did report characteristics for both $\mathrm{RBBB}$ and non-RBBB patients, the median age of the participants was 82.0 IQR (81.4-84.0). The median percentage of men was $49.1 \mathrm{IQR}$ (39.2-58.4). For studies that reported these selected risk factors, the median percentage of hypertension was 76.1 IQR (74.8-81.9), the median percentage of diabetes was 30.2 IQR (28.9-32.8), the median BMI average was 26.7 IQR (24.3-27.1), the median percentage of coronary heart disease was 56.4 IQR (34.4-64.5), the median percentage of heart failure greater than or equal to New York Heart Association Class III was 74.7 IQR (47-77), and the median percentage of chronic obstructive pulmonary disease was 21.6 IQR (19.2-27.7). Current smoking percentage was $20.6 \%$ in the preexisting $\mathrm{RBBB}$ group and $19.9 \%$ in the no RBBB group for the one study that reported this risk factor. Racial characteristics were not reported by the included studies that used separate preexisting RBBB groups. Multiple centers were used by eight of the included studies and Europe, North America, South America, Japan, and Israel were the geographic regions represented.

3.3. PPI in Patients with Preexisting RBBB after TAVR. Auffrett et al., Husser et al., van Gils et al., Tovia-Brodie et al., and Watanabe et al. reported various clinical outcomes in patients with preexisting RBBB after TAVR as summarized in Table 2 [7-11]. Auffrett et al. found patients with preexisting $\mathrm{RBBB}$ to have higher 30 -day PPI rates $(40.1 \%$ vs. 13.5\%; $p<0.001$ ) [7]. Husser et al. reported a 30-day PPI rate of $39.2 \%$ in patients with preexisting RBBB after TAVR and found the ACURATE neo (Boston Scientific, Marlborough, Massachusetts) to have a lower rate of PPI when compared with the Edwards Sapien 3 (Edwards Lifesciences, Irvine, California) [8]. Van Gils et al. reported a 30-day PPI rate of $41.0 \%$ in patients with preexisting RBBB after TAVR and found the Boston Scientific Lotus (Boston Scientific, Marlborough, Massachusetts) to have the highest rate of PPI and the Edwards Sapien 3 and XT (Edwards Lifesciences, Irvine, California) to have the lowest rate of PPI [9]. Watanabe et al. found patients with preexisting $\mathrm{RBBB}$ to have higher 30 -day rates of PPI $(17.6 \%$ vs. $2.9 \%$; $p<0.01)$ [10]. Tovia-Brodie et al. did not report a 30-day PPI rate as they compared whether prophylactic PPI improved outcomes in patients with preexisting RBBB [11].

The nine remaining studies identified risk factors for 30day PPI as summarized in Table 2 [12-20]. Meduri et al. 


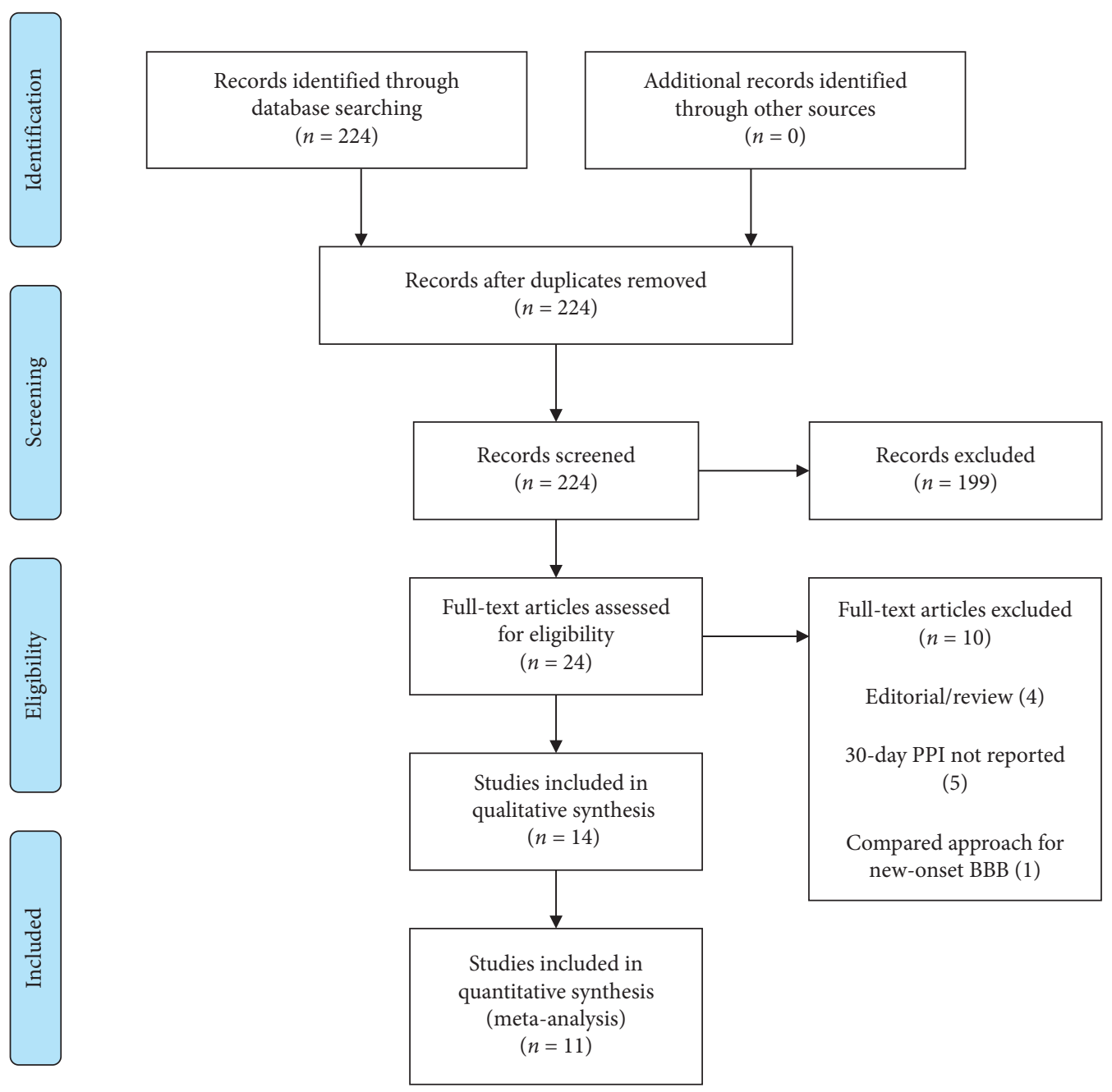

FIgURE 1: Flowchart of the included studies.

identified preexisting RBBB, female sex, and depth of implantation to be a risk factors for 30-day PPI in their retrospective review of the REPRISE III (The Repositionable Percutaneous Replacement of Stenotic Aortic Valve through Implantation of Lotus Valve System-Randomized Clinical Evaluation) trial [12]. Nazif et al. identified preexisting RBBB, prosthesis to left ventricular outflow tract diameter ratio, and left ventricular end-diastolic diameter as risk factors for 30-day PPI in their retrospective review of the PARTNER (Placement of AoRtic TraNscathetER Valves) trial [15]. Dhakal et al., Roten et al., Erkapic et al., Koos et al., and Fraccarro et al. all identified preexisting RBBB as a risk factor for PPI after TAVR in their retrospective single-center studies [13, 16-19]. Bagur et al. found preexisting RBBB as the only risk factor for PPI after TAVR in their comparison to surgical aortic valve replacement [14]. Guetta el al. identified preexisting RBBB and deep valve implantation as risk factors for PPI after TAVR in their retrospective review at three referral centers in Israel [20].

Meta-analysis of the included studies revealed a higher 30-day PPI rate of $38.1 \%$ in patients with preexisting RBBB when compared to a 30 -day PPI rate of $11.4 \%$ in patients with no RBBB. This is a statistically significant increase in 30- day PPI rate in patients with preexisting RBBB with a risk ratio of 3.56 (95\% CI 3.21-3.93, $p<0.01$ ). The forest plot for 30-day PPI rate is shown in Figure 2. Husser et al., ToviaBrodie et al., and van Gils et al. results were not included in the 30-day PPI meta-analysis as these studies only included patients with preexisting RBBB and made no comparisons to patients without RBBB. In the two included studies that reported 30-day mortality as an outcome, meta-analysis revealed a higher 30 -day mortality rate of $9.5 \%$ in patients with preexisting $\mathrm{RBBB}$ compared to a 30 -day mortality rate of $6.3 \%$ in patients with no RBBB. This is a statistically significant increase in 30-day mortality in patients with preexisting RBBB with a risk ratio of 1.60 (95\% CI 1.14-2.25, $p<0.01)$. The forest plot for 30 -day morality rate is shown in Figure 3.

\section{Discussion}

This is the first systematic review and meta-analysis to demonstrate the impact of preexisting RBBB on new pacemaker implantation after TAVR. Our findings are derived from 14 studies reporting clinical outcomes in a total of 1,654 patients with preexisting RBBB after TAVR. The 


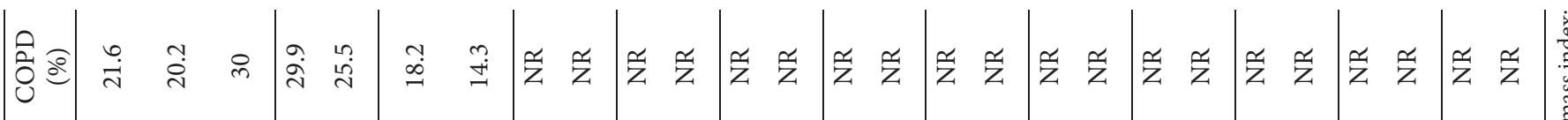

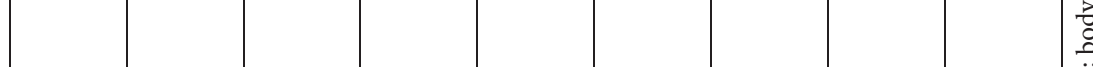

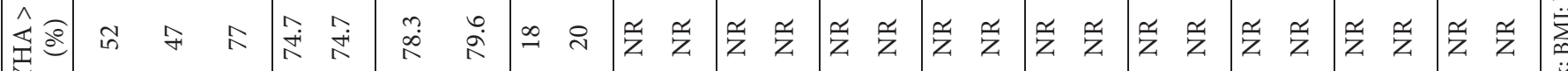

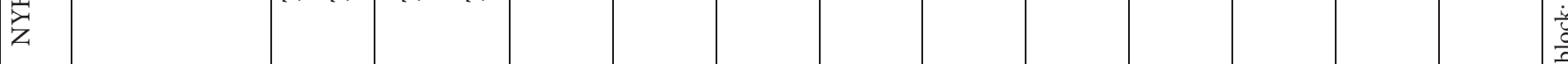

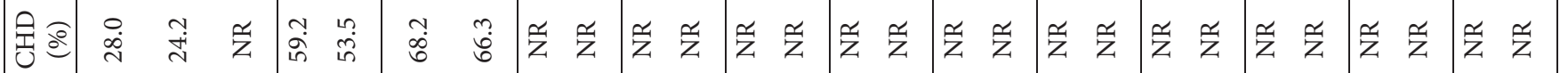

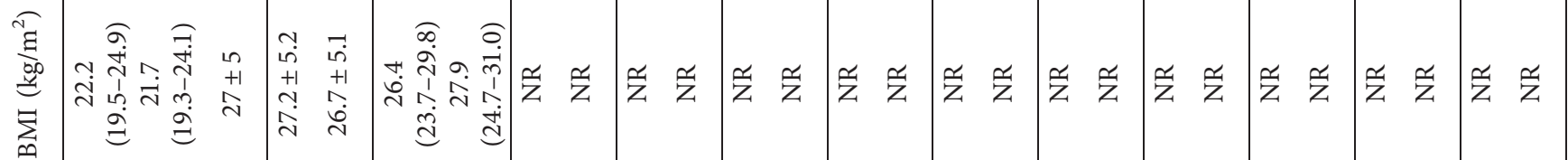

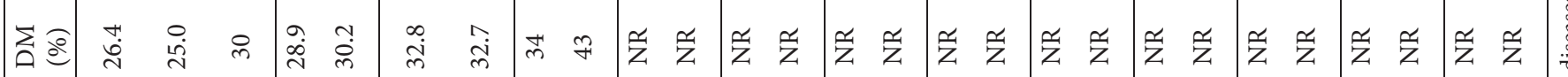

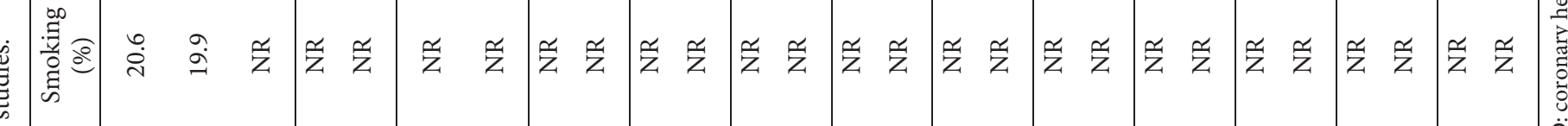

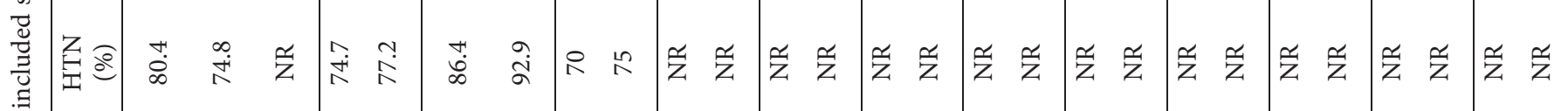

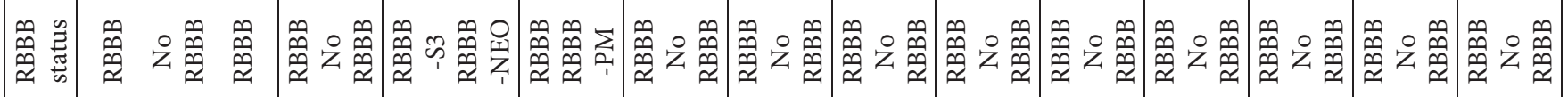

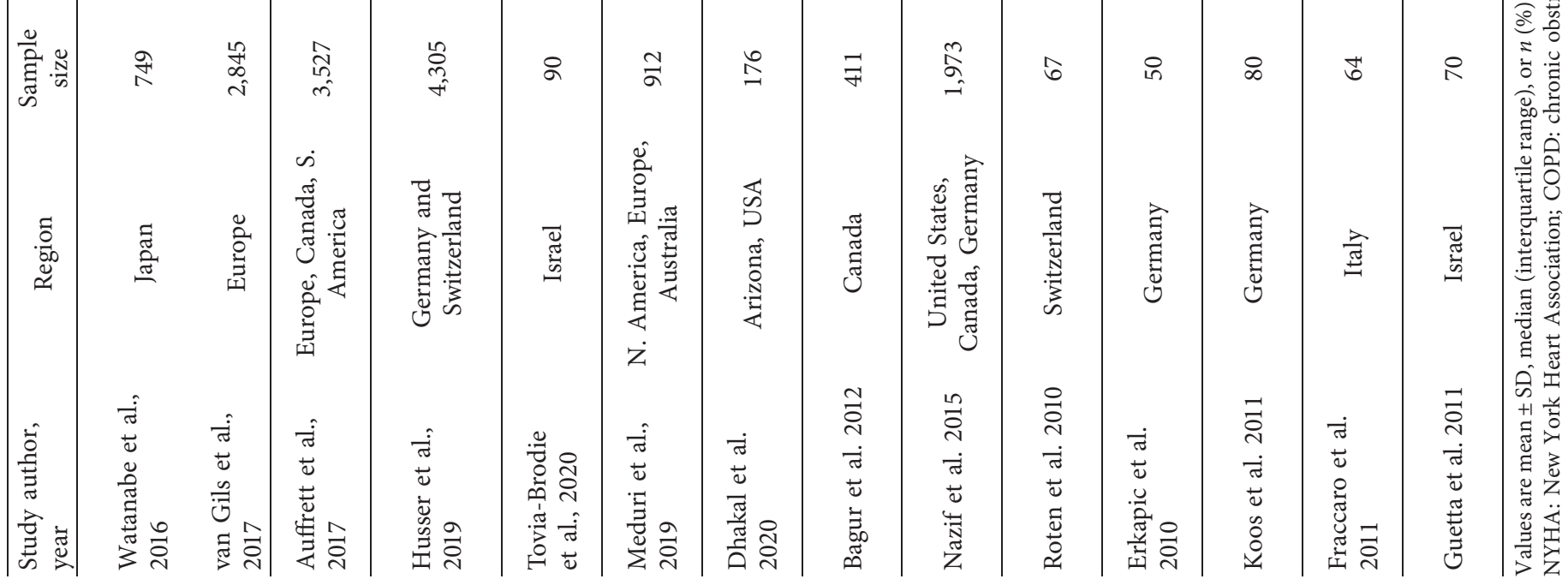


TABLE 2: Outcomes of patients with preexisting RBBB after TAVR summary table.

\begin{tabular}{|c|c|c|c|c|c|c|c|}
\hline References & Year & Region & Centers & $\begin{array}{l}\text { Patients } \\
\text { w/RBBB }\end{array}$ & Valves & Primary outcome & Other outcomes \\
\hline $\begin{array}{l}\text { Watanabe } \\
\text { et al. }\end{array}$ & 2016 & Japan & 8 & 102 & ES-XT & $\begin{array}{c}\text { Various clinical } \\
\text { outcomes }\end{array}$ & PPI, mortality, bleeding, etc. \\
\hline van Gils et al. & 2017 & Europe & 4 & 306 & $\begin{array}{l}\text { CoreValve } \\
\text { ES-XT } \\
\text { ES-3 } \\
\text { Lotus } \\
\end{array}$ & PPI within 30 days & $\begin{array}{l}\text { New onset conduction } \\
\text { disturbances }\end{array}$ \\
\hline Auffrett et al. & 2017 & $\begin{array}{c}\text { Europe, Canada, S. } \\
\text { America }\end{array}$ & $\begin{array}{c}\text { Not } \\
\text { reported }\end{array}$ & 362 & Not reported & All-cause mortality & CV death, SCD, PPI \\
\hline Husser et al. & 2019 & $\begin{array}{l}\text { Germany and } \\
\text { Switzerland }\end{array}$ & 7 & 296 & $\begin{array}{l}\mathrm{Neo} \\
\mathrm{ES}-3\end{array}$ & PPI within 30 days & Device failure \\
\hline $\begin{array}{l}\text { Tovia-Brodie } \\
\text { et al. }\end{array}$ & 2020 & Israel & 1 & 90 & $\begin{array}{c}\text { CoreValve } \\
\text { ES-3 } \\
\text { ES-XT } \\
\text { Evolute R } \\
\text { Lotus } \\
\end{array}$ & $\begin{array}{l}\text { Outcomes comparison } \\
\text { for prophylactic PM }\end{array}$ & Predictors for pacing \\
\hline Meduri et al. & 2019 & $\begin{array}{l}\text { N. America, } \\
\text { Europe, Australia }\end{array}$ & 55 & 85 & $\begin{array}{l}\text { CoreValve } \\
\text { Lotus }\end{array}$ & PPI within 30 days & $\begin{array}{l}\text { Predictors for pacing, mortality, } \\
\text { stroke, rehospitalization }\end{array}$ \\
\hline Dhakal et al. & 2020 & Arizona, USA & 1 & 36 & $\begin{array}{c}\text { Not reported, balloon } \\
\text { expandable and self- } \\
\text { expanding }\end{array}$ & PPI within 30 days & Predictors for pacing \\
\hline Bagur et al. & 2012 & Canada & 3 & 20 & $\begin{array}{c}\text { CE } \\
\text { ES } \\
\text { ES-XT } \\
\end{array}$ & PPI within 30 days & Predictors for pacing \\
\hline Naziif et al. & 2015 & $\begin{array}{c}\text { United States, } \\
\text { Canada, Germany } \\
\end{array}$ & 21 & 312 & ES & PPI within 30 days & Predictors for pacing \\
\hline Roten et al. & 2010 & Switzerland & 1 & 13 & $\begin{array}{c}\text { CoreValve } \\
\text { ES }\end{array}$ & PPI within 30 days & Predictors for pacing \\
\hline Erkapic et al. & 2010 & Germany & 1 & 7 & $\begin{array}{l}\text { CoreValve } \\
\text { ES }\end{array}$ & PPI within 30 days & Predictors for pacing \\
\hline Koos et al. & 2011 & Germany & 1 & 6 & $\begin{array}{l}\text { CoreValve } \\
\text { ES }\end{array}$ & PPI within 30 days & Predictors for pacing \\
\hline Fraccaro et al. & 2011 & Italy & 1 & 8 & CoreValve & PPI within 30 days & Predictors for pacing \\
\hline Guetta et al. & 2011 & Israel & 3 & 11 & CoreValve & PPI within 30 days & Predictors for pacing \\
\hline
\end{tabular}

PPI: permanent pacemaker implantation; SCD: sudden cardiac death; CV: cardiovascular; ES-XT: Edwards SAPIEN XT; ES-3: Edwards SAPIEN 3; ES: Edwards SAPIEN; CE: Cribier-Edwards.

incidence of new PPI was significantly increased in patients with preexisting RBBB after TAVR. Increased all-cause and cardiovascular mortality has been demonstrated in patients with preexisting RBBB after TAVR.

The prognostic value of RBBB has shown mixed results in previous studies with healthy participants and with patients with heart disease [21-28]. Bussink et al. found RBBB to be associated with increased all-cause and cardiovascular mortality in both men and women from the general population [21]. Abdel-Qadir et al. found no prognostic value of RBBB in patients hospitalized with heart failure; however, Barshehet et al. found RBBB to be associated with increased long-term mortality risk in hospitalized patients with systolic heart failure [22, 23]. Melgaregjo-Moreno et al. found new permanent RBBB to be associated with increased 30-day and seven-year mortality in patients with acute myocardial infarction [24]. Wong et al. found RBBB accompanying anterior acute myocardial infarction to be associated with increased 30-day mortality [28]. Long-term epidemiological studies in men from the general population have found a higher incidence of RBBB with aging, hypertension, and diabetes mellitus $[25,26]$. Zhang et al. found RBBB in women with cardiovascular disease to be associated with an increased risk of coronary heart disease death over 14 years of follow-up. However, RBBB in women free of cardiovascular disease was not associated with increased mortality [27]. Meta-analysis completed by Xiong et al. found RBBB to be associated with an increased risk of mortality in the general population and in patients with heart disease [6]. The exact mechanism by which RBBB increases mortality has not been elucidated, although underlying conduction system disease can predispose patients to various arrhythmias. The association of RBBB with decreased left ventricular ejection fraction in patients with prior myocardial infarction or heart failure may provide a clue towards the underlying mechanism $[23,28]$. The various comorbidities and underlying heart disease in patients with RBBB may also explain the increased mortality.

A previous meta-analysis by Siontis et al. demonstrated the significance of RBBB in the requirement for PPI after TAVR. Male sex, first-degree AV block, left anterior hemiblock, and intraprocedural AV block were also found to be predictive of PPI after TAVR [4]. The newer studies highlighted in this meta-analysis emerged to specifically focus on the clinical impact of preexisting RBBB after TAVR, which is important for improving patient outcomes [7-10]. 


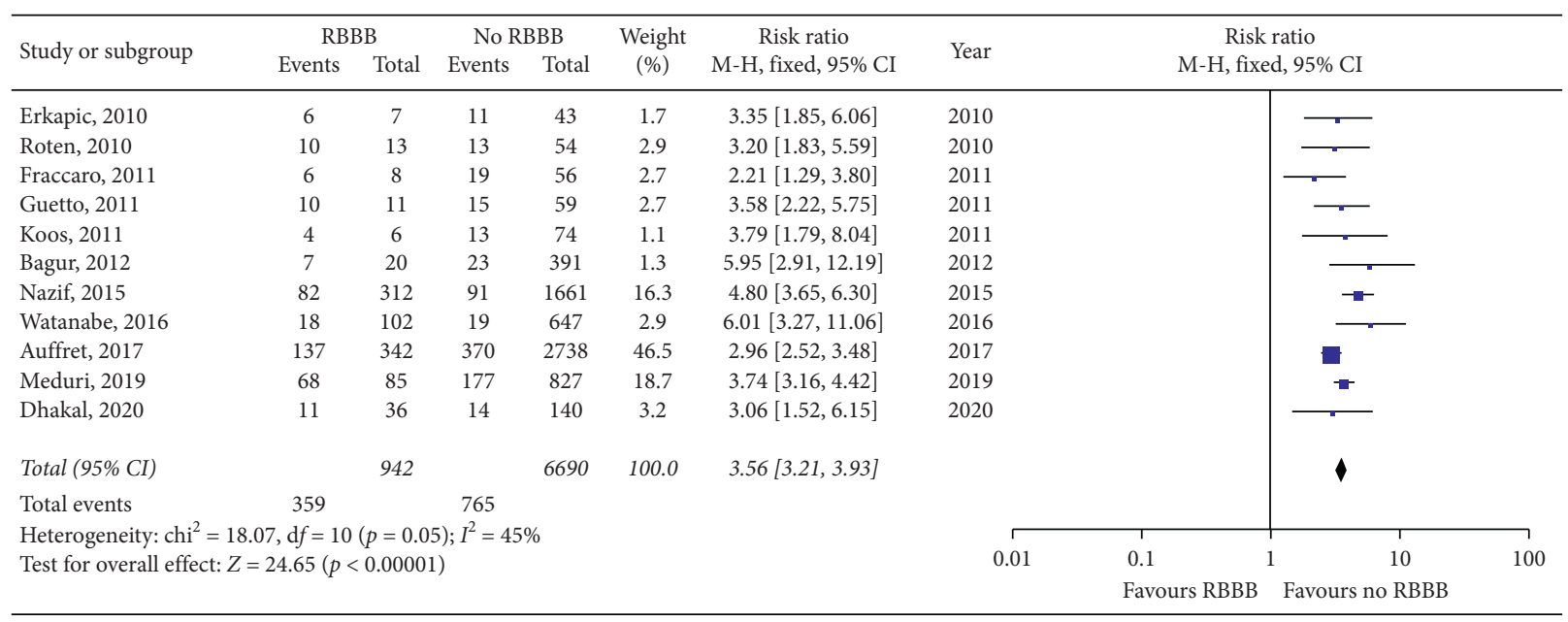

FIGURE 2: Forest plot of 30-day PPI rates in patients with and without preexisting RBBB after TAVR.

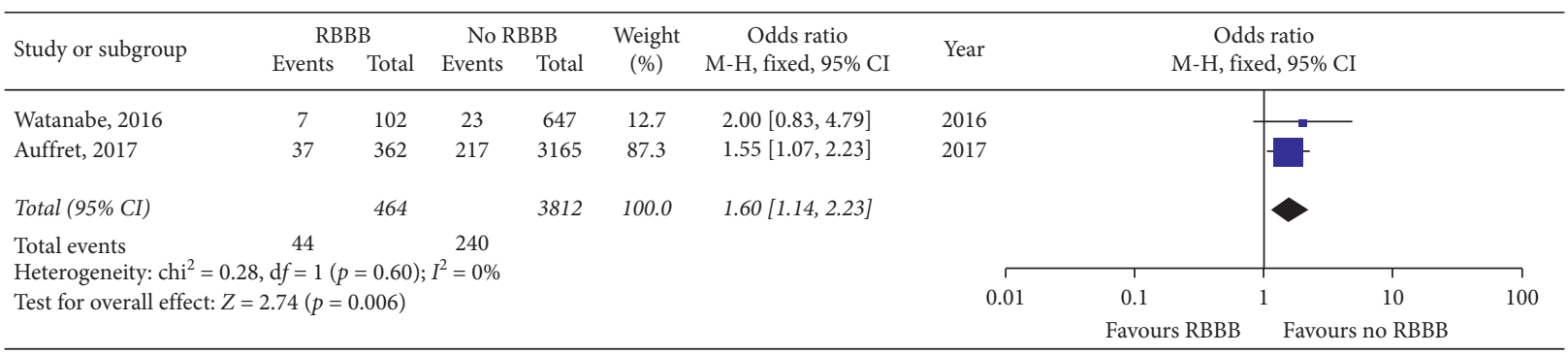

FIgURE 3: Forest plot of 30-day all-cause mortality rate in patients with and without preexisting RBBB after TAVR.

In TAVR, conduction disturbances are a common complication likely due to direct insult to the left bundle branch because of the anatomical relationship between the aortic annulus and the conduction system [29]. New-onset LBBB develops in 5\% to $65 \%$ of patients undergoing TAVR, and its persistence can result in PPI in $15 \%$ to $20 \%$ of cases [29]. New-onset LBBB after TAVR has been associated with increased risk of cardiac death and PPI at one year follow-up [30]. Preexisting RBBB with new-onset LBBB after TAVR will usually generate PPI during the index hospitalization. Chorianopoulos et al. demonstrated that postprocedural bradyarrhythmias develop in $36.2 \%$ of patients after TAVR with $3.8 \%$ remaining $>96$ hours after TAVR. Preexisting $\mathrm{RBBB}$ was found to be the only predictor of postprocedural bradyarrhythmias [5]. Late-onset new LBBB $>3$ months after TAVR is a rare complication seen in only $0.8 \%$ of patients [31]. Development of high degree AV block is a common complication seen in up to $58.8 \%$ of patients with preexisting $\mathrm{BBB}$ or $\mathrm{BBB}$ occurring during TAVR [32]. These late conduction disorders in patients with preexisting RBBB can lead to cardiac complications such as heart failure and sudden cardiac death.

This systematic review and meta-analysis reveals increased PPI, all-cause mortality, and cardiac mortality in patients with preexisting RBBB after TAVR. This is clinically significant given the recent trend towards early discharge after TAVR [33]. Patients with preexisting RBBB may need additional monitoring after TAVR to detect conduction disturbances and ensure safe discharge. Early electrocardiographic monitoring may be beneficial as part of the TAVR work-up as Urena et al. found that newly diagnosed preprocedural arrhythmias are common and associated with higher rates of PPI after the procedure [34]. Additional strategies for managing preexisting $\mathrm{RBBB}$ in patients undergoing TAVR may emerge as we understand more about conduction disturbances following TAVR.

Preexisting $\mathrm{RBBB}$ is a common underlying conduction disturbance in patients undergoing TAVR and is associated with increased risk of PPI at 30 days and all-cause and cardiovascular mortality. Future studies will be needed to evaluate optimal management of patients with preexisting RBBB undergoing TAVR. Larger prospective studies are needed to investigate the optimal timing for PPI after TAVR and to evaluate prophylactic PPI in patients with preexisting RBBB prior to TAVR. Larger prospective studies are needed to investigate strategies for early detection of conduction disturbances in patients with preexisting RBBB. Until more data is available, there are many multicenter and literaturebased decisional algorithms to guide PPI decision-making [35]. Careful monitoring to detect arrhythmias after TAVR may be necessary to improve clinical outcomes in patients with preexisting $\mathrm{RBBB}$.

\section{Limitations}

The limitations for this systematic review and meta-analysis are influenced by the limitations of the included studies. Auffrett et al. used a nonrandomized study design that may 
lead to confounders influencing the relationship between preexisting RBBB and outcomes [7]. The studies by Husser et al. and van Gils et al. are limited by their observational design $[8,9]$. Tovia-Brodie et al. used a single-center retrospective design and did not use randomization to determine prophylactic pacemaker implantation [11]. The results from Watanabe et al. are limited by the relatively small size of the cohort $(n=749)$ and the relatively short median follow-up of 16 months [10]. The retrospective studies of existing data are subject to all of the limitations inherent to this study design [12-20]. Availability of specific data points, such as medication that could influence cardiac conduction, is a common limitation for retrospective studies. Roten et al., Erkapic et al., Koos et al., Fraccaro et al., and Guetta et al. all had small sample sizes of less than 100 patients in their studies [16-20]. All of the included studies are likely influenced by between-center variability and the lack of centralized independent assessment of procedural results and outcomes. The various valve types used in each study likely influence the generalizability of the aggregate data as specific valve types have shown different rates of procedural complications.

\section{Conclusion}

Conduction issues after TAVR continue to remain a common complication during the management of severe aortic stenosis. This current systematic review and meta-analysis indicates that patients with preexisting RBBB have a higher incidence of PPI and all-cause mortality after 30 days after TAVR compared with patients without RBBB. Further trials are needed to compare the clinical outcomes based on TAVR valve types and assess the benefit of PPI in patients with RBBB after TAVR. In addition, understanding the progression and prevention of electrical conduction disease are necessary for appropriate risk stratification, interventional strategy, and avoidance of pacemaker implantation.

\section{Data Availability}

The data used to support the findings of this study are included within the article.

\section{Conflicts of Interest}

The authors declare no conflicts of interest.

\section{References}

[1] A. Slomski, "CBT for Body dysmorphic disorder," JAMA, vol. 321, no. 20, p. 1965, 2019.

[2] S. V. Arnold, Y. Zhang, S. J. Baron et al., "Impact of short-term complications on mortality and quality of life after transcatheter aortic valve replacement," JACC: Cardiovascular Interventions, vol. 12, no. 4, pp. 362-369, 2019.

[3] M. Urena and J. Rodés-Cabau, "Conduction abnormalities," JACC: Cardiovascular Interventions, vol. 9, no. 21, pp. 22172219, 2016.

[4] G. C. M. Siontis, P. Jüni, T. Pilgrim et al., "Predictors of permanent pacemaker implantation in patients with severe aortic stenosis undergoing TAVR," Journal of the American College of Cardiology, vol. 64, no. 2, pp. 129-140, 2014.

[5] E. Chorianopoulos, U. Krumsdorf, S. T. Pleger, H. A. Katus, and R. Bekeredjian, "Incidence of late occurring bradyarrhythmias after TAVI with the self-expanding CoreValve aortic bioprosthesis," Clinical Research in Cardiology, vol. 101, no. 5, pp. 349-355, 2012.

[6] Y. Xiong, L. Wang, W. Liu, G. J. Hankey, B. Xu, and S. Wang, "The prognostic significance of right bundle branch block: a meta-analysis of prospective cohort studies," Clinical Cardiology, vol. 38, no. 10, pp. 604-613, 2015.

[7] V. Auffret, J. G. Webb, H. Eltchaninoff et al., "Clinical impact of baseline right bundle branch block in patients undergoing transcatheter aortic valve replacement," JACC. Cardiovascular Interventions, vol. 10, no. 15, pp. 1564-1574, 2017.

[8] O. Husser, C. Pellegrini, W.-K. Kim et al., "Transcatheter valve SELECTion in patients with right bundle branch block and impact on pacemaker implantations," JACC: Cardiovascular Interventions, vol. 12, no. 18, pp. 1781-1793, 2019.

[9] L. van Gils, D. Tchetche, T. Lhermusier et al., "Transcatheter heart valve selection and permanent pacemaker implantation in patients with pre-existent right bundle branch block," Journal of the American Heart Association, vol. 6, no. 3, 2017.

[10] Y. Watanabe, K. Kozuma, H. Hioki et al., "Pre-existing right bundle branch block increases risk for death after transcatheter aortic valve replacement with a balloon-expandable valve," JACC: Cardiovascular Interventions, vol. 9, no. 21, pp. 2210-2216, 2016.

[11] O. Tovia-Brodie, S. L. Shesaf, A. Hochstadt et al., "The utility of prophylactic pacemaker implantation in right bundle branch block patients pre-transcatheter aortic valve implantation," The Israel Medical Association Journal, vol. 12, no. 21, pp. 790-795, 2019.

[12] C. U. Meduri, D. J. Kereiakes, V. Rajagopal et al., "Pacemaker implantation and dependency after transcatheter aortic valve replacement in the REPRISE III trial," Journal of the American Heart Association, vol. 8, no. 21, 2019.

[13] B. P. Dhakal, K. A. Skinner, K. Kumar et al., "Arrhythmias in relation to mortality after transcatheter aortic valve replacement," The American Journal of Medicine, 2020.

[14] R. Bagur, J. Rodés-Cabau, R. Gurvitch et al., "Need for permanent pacemaker as a complication of transcatheter aortic valve implantation and surgical aortic valve replacement in elderly patients with severe aortic stenosis and similar baseline electrocardiographic findings," JACC: Cardiovascular Interventions, vol. 5, no. 5, pp. 540-551, 2012.

[15] T. M. Nazif, J. M. Dizon, R. T. Hahn et al., "Predictors and clinical outcomes of permanent pacemaker implantation after transcatheter aortic valve replacement: the PARTNER (Placement of AoRtic TraNscathetER Valves) trial and registry," JACC. Cardiovascular Interventions, vol. 8, no. 1 Pt A, pp. 60-69, 2015.

[16] L. Roten, P. Wenaweser, E. Delacrétaz et al., "Incidence and predictors of atrioventricular conduction impairment after transcatheter aortic valve implantation," The American Journal of Cardiology, vol. 106, no. 10, pp. 1473-1480, 2010.

[17] D. Erkapic, W. K. Kim, M. Weber et al., "Electrocardiographic and further predictors for permanent pacemaker requirement after transcatheter aortic valve implantation," Europace, vol. 12, no. 8, pp. 1188-1190, 2010.

[18] R. Koos, A. H. Mahnken, O. Aktug et al., "Electrocardiographic and imaging predictors for permanent pacemaker requirement after transcatheter aortic valve implantation," 
The Journal of Heart Valve Disease, vol. 20, no. 1, pp. 83-90, 2011.

[19] C. Fraccaro, G. Buja, G. Tarantini et al., "Incidence, predictors, and outcome of conduction disorders after transcatheter selfexpandable aortic valve implantation," The American Journal of Cardiology, vol. 107, no. 5, pp. 747-754, 2011.

[20] V. Guetta, G. Goldenberg, A. Segev et al., "Predictors and course of high-degree atrioventricular block after transcatheter aortic valve implantation using the CoreValve Revalving System," The American Journal of Cardiology, vol. 108, no. 11, pp. 1600-1605, 2011.

[21] B. E. Bussink, A. G. Holst, L. Jespersen, J. W. Deckers, G. B. Jensen, and E. Prescott, "Right bundle branch block: prevalence, risk factors, and outcome in the general population: results from the Copenhagen City Heart Study," European Heart Journal, vol. 34, no. 2, pp. 138-146, 2013.

[22] H. M. Abdel-Qadir, J. V. Tu, P. C. Austin, J. T. Wang, and D. S. Lee, "Bundle branch block patterns and long-term outcomes in heart failure," International Journal of Cardiology, vol. 146, no. 2, pp. 213-218, 2011.

[23] A. Barsheshet, I. Goldenberg, M. Garty et al., "Relation of bundle branch block to long-term (four-year) mortality in hospitalized patients with systolic heart failure," The American Journal of Cardiology, vol. 107, no. 4, pp. 540-544, 2011.

[24] A. Melgarejo-Moreno, J. Galcerá-Tomás, L. ConsuegraSánchez et al., "Relation of new permanent right or left bundle branch block on short- and long-term mortality in acute myocardial infarction bundle branch block and myocardial infarction," The American Journal of Cardiology, vol. 116, no. 7, pp. 1003-1009, 2015.

[25] I. S. Thrainsdottir, T. Hardarson, G. Thorgeirsson, H. Sigvaldason, and N. Sigfusson, "The epidemiology of right bundle branch block and its association with cardiovascular morbidity-the Reykjavik Study," European Heart Journal, vol. 14, no. 12, pp. 1590-1596, 1993.

[26] P. Eriksson, P.-O. Hansson, H. Eriksson, and M. Dellborg, "Bundle-branch block in a general male population," Circulation, vol. 98, no. 22, pp. 2494-2500, 1998.

[27] Z.-m. Zhang, P. M. Rautaharju, E. Z. Soliman et al., "Mortality risk associated with bundle branch blocks and related repolarization abnormalities (from the Women's Health Initiative [WHI])," The American Journal of Cardiology, vol. 110, no. 10, pp. 1489-1495, 2012.

[28] C.-K. Wong, R. A. H. Stewart, W. Gao, J. K. French, C. Raffel, and H. D. White, "Prognostic differences between different types of bundle branch block during the early phase of acute myocardial infarction: insights from the Hirulog and Early Reperfusion or Occlusion (HERO)-2 trial," European Heart Journal, vol. 27, no. 1, pp. 21-28, 2006.

[29] G. Massoullié, P. Bordachar, K. A. Ellenbogen et al., "Newonset left bundle branch block induced by transcutaneous aortic valve implantation," The American Journal of Cardiology, vol. 117, no. 5, pp. 867-873, 2016.

[30] A. Regueiro, O. A.-J. Altisent, M. D. Trigo et al., "Impact of new-onset left bundle branch block and periprocedural permanent pacemaker implantation on clinical outcomes in patients undergoing transcatheter aortic valve replacement," Circulation: Cardiovascular Interventions, vol. 9, no. 5, 2016.

[31] P. Houthuizen, R. M. A. van der Boon, M. Urena et al., "Occurrence, fate and consequences of ventricular conduction abnormalities after transcatheter aortic valve implantation," EuroIntervention, vol. 9, no. 10, pp. 1142-1150, 2014.

[32] F. Egger, M. Nürnberg, M. Rohla et al., "High-degree atrioventricular block in patients with preexisting bundle branch block or bundle branch block occurring during transcatheter aortic valve implantation," Heart Rhythm, vol. 11, no. 12, pp. 2176-2182, 2014.

[33] E. Durand, H. Eltchaninoff, A. Canville et al., "Feasibility and safety of early discharge after transfemoral transcatheter aortic valve implantation with the Edwards SAPIEN-XT prosthesis," The American Journal of Cardiology, vol. 115, no. 8, pp. 1116-1122, 2015.

[34] M. Urena, S. Hayek, A. N. Cheema et al., "Arrhythmia burden in elderly patients with severe aortic stenosis as determined by continuous electrocardiographic recording," Circulation, vol. 131, no. 5, pp. 469-477, 2015.

[35] V. Auffret, R. Puri, M. Urena et al., "Conduction disturbances after transcatheter aortic valve replacement," Circulation, vol. 136, no. 11, pp. 1049-1069, 2017. 